PROCEEDINGS OF THE

AMERICAN MATHEMATICAL SOCIETY

Volume 132, Number 11, Pages 3289-3298

S 0002-9939(04)07571-9

Article electronically published on June 18, 2004

\title{
ALMOST AUTOMORPHIC SOLUTIONS OF EVOLUTION EQUATIONS
}

\author{
TOKA DIAGANA, GASTON NGUEREKATA, AND NGUYEN VAN MINH \\ (Communicated by Carmen C. Chicone)
}

\begin{abstract}
This paper is concerned with the existence of almost automorphic mild solutions to equations of the form

(*)

$$
\dot{u}(t)=A u(t)+f(t),
$$

where $A$ generates a holomorphic semigroup and $f$ is an almost automorphic function. Since almost automorphic functions may not be uniformly continuous, we introduce the notion of the uniform spectrum of a function. By modifying the method of sums of commuting operators used in previous works for the case of bounded uniformly continuous solutions, we obtain sufficient conditions for the existence of almost automorphic mild solutions to $(*)$ in terms of the imaginary spectrum of $A$ and the uniform spectrum of $f$.
\end{abstract}

\section{INTRODUCTION AND NOTATION}

In this paper we deal with the existence of almost automorphic mild solutions to evolution equations of the form

$$
\frac{d u}{d t}=A u+f(t)
$$

where $A$ is a (unbounded) linear operator that generates a holomorphic semigroup of linear operators on a Banach space $\mathbb{X}$ and $f$ is an almost automorphic function taking values in $\mathbb{X}$.

This problem has been of great interest to many mathematicians for decades. Actually, it goes back to the characterization of exponential dichotomy of linear ordinary differential equations by $\mathrm{O}$. Perron. The reader can find many extensions of the classical result of Perron to the infinite-dimensional case in [4, 11, 15, 17, 27. and the references therein with results concerned with almost periodic solutions and bounded solutions. Recently, the interest in finding conditions for the existence of automorphic solutions has been regained (see, e.g., [21, 26]). Some extensions of results on almost periodic solutions have been made in [10]. In this direction, we study conditions for the existence and uniqueness of almost automorphic solutions to Eq. (1). The idea of using the method of sums of commuting operators to study the existence of almost periodic solutions is due to Murakami, Naito and Minh [15]. This method works smoothly in the framework of evolution semigroups

Received by the editors July 16, 2003.

2000 Mathematics Subject Classification. Primary 34G10; Secondary 43A60.

Key words and phrases. Analytic semigroup, almost automorphic solution, uniform spectrum, sums of commuting operators. 
associated with evolution equations. However, in our problem setting, in general, the associated evolution semigroups are not strongly continuous since almost automorphic functions are not necessarily uniformly continuous. Other methods of proving the existence of bounded and uniformly continuous solutions in [23, 27] are inapplicable due to the explicit use of uniform continuity. The main task in this paper is to overcome this difficulty to prove a necessary and sufficient condition for the existence and uniqueness of almost automorphic mild solutions of the form $\sigma(A) \cap i s p(f)=\oslash$. To this end, we introduce the notion of uniform spectrum of a function, which turns out to be appropriate for extending the method of sums of commuting operators to the case of non-uniform continuity. Our main results obtained in this paper are Theorem 4.5 and Corollary 4.6

Notation. Throughout the paper, $\mathbb{R}, \mathbb{C}, \mathbb{X}$ stand for the sets of real, complex numbers and a complex Banach space, respectively; $L(\mathbb{X}), B C(\mathbb{R}, \mathbb{X}), B U C(\mathbb{R}, \mathbb{X})$, $A P(\mathbb{X})$ denote the spaces of linear bounded operators on $\mathbb{X}$, all $\mathbb{X}$-valued bounded continuous functions, all $\mathbb{X}$-valued bounded uniformly continuous functions and almost periodic functions in Bohr's sense (see [14 p. 4]) with sup-norm, respectively.

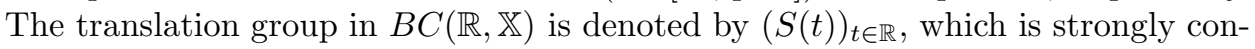
tinuous in $B U C(\mathbb{R}, \mathbb{X})$ and whose infinitesimal generator is the differential operator $d / d t$. For a linear operator $A$, we denote by $D(A), \sigma(A)$ and $\rho(A)$ the domain, spectrum and resolvent set of $A$, respectively. If $Y$ is a metric space and $B$ is a subset of $Y$, then $\bar{B}$ denotes its closure in $Y$. In this paper by the notion of sectorial operators is meant the one defined in [22]. The notion of closure of an operator is referred to the one defined in 6 .

\section{Spectral THEORY OF FUnCtions}

2.1. Spectrum of a function in $B C(\mathbb{R}, \mathbb{X})$. In the present paper, for $u \in$ $B C(\mathbb{R}, \mathbb{X}), s p(u)$ stands for the Carleman spectrum, which consists of all $\xi \in \mathbb{R}$ such that the Carleman-Fourier transform of $u$, defined by

$$
\hat{u}(\lambda):= \begin{cases}\int_{0}^{\infty} e^{-\lambda t} u(t) d t & (\operatorname{Re} \lambda>0), \\ -\int_{0}^{\infty} e^{\lambda t} u(-t) d t & (\operatorname{Re} \lambda<0),\end{cases}
$$

has no holomorphic extension to any neighborhoods of $i \xi$ (see [23. Prop. 0.5, p. $22])$. For each $u \in B C(\mathbb{R}, \mathbb{X})$ we denote $\mathcal{M}_{u}:=\overline{\operatorname{span}\{S(\tau) u, \tau \in \mathbb{R}\}}$, which is a closed subspace of $B C(\mathbb{R}, \mathbb{X})$. If $u \in B U C(\mathbb{R}, \mathbb{X})$, the Carleman spectrum of $u$ coincides with its Arveson spectrum, defined by (see [2, Lemma 4.6.8])

$$
i s p(u)=\sigma\left(\mathcal{D}_{u}\right),
$$

where $\mathcal{D}_{u}$ is the infinitesimal generator of the restriction of the group of translations $\left(\left.S(t)\right|_{\mathcal{M}_{u}}\right)_{t \in \mathbb{R}}$ to the closed subspace $\mathcal{M}_{u}$.

Below we list some properties of the spectra of functions which we will need in the sequel.

Proposition 2.1. Let $u, u_{n}, v \in B C(\mathbb{R}, \mathbb{X})$ be such that $\lim _{n \rightarrow \infty}\left\|u_{n}-u\right\|=0$, and $\psi \in \mathcal{S}$. Then

(i) $s p(u)$ is closed,

(ii) $s p(u+v) \subset \operatorname{sp}(u) \cup \operatorname{sp}(v)$,

(iii) $s p(\psi * u) \subset \operatorname{sp}(u) \cap \operatorname{supp} \tilde{\psi}$, 
(iv) $s p(u-\psi * u) \subset s p(u) \cap \operatorname{supp}(1-\tilde{\psi})$,

(v) if $\tilde{\psi} \equiv 1$ on a neighborhood of $\operatorname{sp}(u)$, then $\psi * u=u$,

(vi) if $\operatorname{sp}(u) \cap \operatorname{supp} \tilde{\psi}=\emptyset$, then $\psi * u=0$,

(vii) if $s p\left(u_{n}\right) \subset \Lambda, \forall n$, then $s p(u) \subset \bar{\Lambda}$.

Proof. We refer the reader to 23, Prop. 0.4, Prop. 0.6, Theorem 0.8, pp. 2025].

2.2. Uniform spectrum of a function in $B C(\mathbb{R}, \mathbb{X})$. Notice that for every $\lambda \in \mathbb{C}$ with $\Re \lambda \neq 0$ and $f \in B C(\mathbb{R}, \mathbb{X})$ the function $\varphi_{f}(\lambda): \mathbb{R} \ni t \mapsto \widehat{S(t) f}(\lambda) \in \mathbb{X}$ belongs to $\mathcal{M}_{f} \subset B C(\mathbb{R}, \mathbb{X})$. Moreover, $\varphi_{f}(\lambda)$ is analytic on $\mathbb{C} \backslash i \mathbb{R}$.

Definition 2.2. Let $f$ be in $B C(\mathbb{R}, \mathbb{X})$. Then,

(i) $\alpha \in \mathbb{R}$ is said to be uniformly regular with respect to $f$ if there exists a neighborhood $\mathcal{U}$ of $i \alpha$ in $\mathbb{C}$ such that the function $\varphi_{f}(\lambda)$, as a complex function of $\lambda$ with $\Re \lambda \neq 0$, has an analytic continuation into $\mathcal{U}$.

(ii) The set of $\xi \in \mathbb{R}$ such that $\xi$ is not uniform regular with respect to $f \in$ $B C(\mathbb{R}, \mathbb{X})$ is called the uniform spectrum of $f$ and is denoted by $s p_{u}(f)$.

If $f \in B U C(\mathbb{R}, \mathbb{X})$, then $\alpha \in \mathbb{R}$ is uniformly regular if and only if it is regular with respect to $f$. In fact, this follows from the fact that for bounded uniformly continuous functions $u$ we have

$$
i s p(u)=\sigma\left(\mathcal{D}_{u}\right) .
$$

Next, using the identity

$$
R\left(\lambda, \mathcal{D}_{u}\right) u=\int_{0}^{\infty} e^{-(\lambda) \xi} S(\xi) u d \xi, \quad \Re \lambda \neq 0
$$

we get the claim. For $f \in B C(\mathbb{R}, \mathbb{X})$, in general, the above (2) may not hold. We now study properties of uniform spectra of functions in $B C(\mathbb{R}, \mathbb{X})$.

Proposition 2.3. Let $g, f, f_{n} \in B C(\mathbb{R}, \mathbb{X})$ be such that $f_{n} \rightarrow f$ as $n \rightarrow \infty$, and let $\Lambda \subset \mathbb{R}$ be a closed subset. Then the following assertions hold:

(i) $s p_{u}(f)=s p_{u}(f(h+\cdot))$;

(ii) $s p_{u}(\alpha f(\cdot)) \subset s p_{u}(f), \alpha \in \mathbb{C}$;

(iii) $s p(f) \subset s p_{u}(f)$;

(iv) $s p_{u}(B f(\cdot)) \subset s p_{u}(f), B \in L(\mathbb{X})$;

(v) $s p_{u}(f+g) \subset s p_{u}(f) \cup s p_{u}(g)$;

(vi) $s p_{u}(f) \subset \Lambda$.

Proof. (i) - (v) are obvious from the definitions of spectrum and uniform spectrum. Now we prove (vi). This can be done by following the proof of the similar assertion for the notion of Carleman spectrum (see for instance [23, Theorem 0.8, p. 21]). For the reader's convenience we reproduce it below.

Let $\rho_{0} \notin \Lambda$. Since $\Lambda$ is closed, there is a positive constant $r<\operatorname{dist}\left(\rho_{0}, \Lambda\right)$. As in the proof of [23, Theorem 0.8, p. 21] or by [2, Lemma 4.6.6, p. 295] we can prove that since

$$
\left\|\varphi_{f_{n}}(\lambda)\right\| \leq \frac{2\|f\|}{|\Re \lambda|}, \quad \forall \lambda \in \bar{B}_{r}\left(i \rho_{0}\right)
$$

for sufficiently large $n \geq N$, one has

$$
\left\|\varphi_{f_{n}}(\lambda)\right\| \leq \frac{4\|f\|}{3 r}, \quad \forall \lambda \in \bar{B}_{r}\left(i \rho_{0}\right), n \geq N .
$$


Obviously, for every fixed $\lambda$ such that $\Re \lambda \neq 0$ we have $\varphi_{f_{n}}(\lambda) \rightarrow \varphi_{f}(\lambda)$. Now applying Vitali's theorem to the sequence of complex functions $\left\{\varphi_{f_{n}}\right\}$ we see that $\varphi_{f_{n}}$ is convergent uniformly on $B_{r}\left(i \rho_{0}\right)$ to $\varphi_{f}$. This yields that $\varphi_{f}$ is holomorphic on $B_{r}\left(i \rho_{0}\right)$, that is, $\rho_{0}$ is a uniform regular point with respect to $f$ and $\rho_{0} \notin s p_{u}(f)$.

As an immediate consequence of (iii) of the above proposition, we have

Corollary 2.4. For any closed subset $\Lambda \subset \mathbb{R}$, the set $\Lambda_{u}(\mathbb{X}):=\{f \in B C(\mathbb{R}, \mathbb{X})$ : $\left.s p_{u}(f) \subset \Lambda\right\}$ is a closed subspace of $B C(\mathbb{R}, \mathbb{X})$ that is invariant under translations.

The following result will be needed in the sequel and is of independent interest.

Lemma 2.5. Let $\Lambda$ be a closed subset of $\mathbb{R}$, and let $\mathcal{D}_{\Lambda_{u}}$ be the differential operator acting on $\Lambda_{u}(\mathbb{X})$. Then we have

$$
\sigma\left(\mathcal{D}_{\Lambda_{u}}\right)=i \Lambda
$$

Proof. Since the function $g_{\alpha}$ defined by $g_{\alpha}(t):=e^{i \alpha t} x, \quad \alpha \in \mathbb{R}, t \in \mathbb{R}, x \neq 0$, is in $\Lambda_{u}(\mathbb{X})$ and $s p_{u}\left(g_{\alpha}\right)=s p\left(g_{\alpha}\right)=\{\alpha\}$ we see that $i \alpha \in \sigma\left(\mathcal{D}_{\Lambda_{u}}\right)$, that is, $i \Lambda \subset \sigma\left(\mathcal{D}_{\Lambda_{u}}\right)$. Now we prove the converse. For $\beta \in \mathbb{R} \backslash \Lambda$ we consider the equation

$$
i \beta g-g^{\prime}=f, \quad f \in \Lambda_{u}(\mathbb{X}) .
$$

We will prove that (6) is uniquely solvable for every $f \in \Lambda_{u}(\mathbb{X})$. This equation has at most one solution. In fact, if $g_{1}, g_{2}$ are two solutions, then $g=g_{1}-g_{2}$ is a solution of the homogeneous equation, that is, for $f=0$. Taking the Carlemann transform of both sides of the corresponding equation we may see that $s p(g) \subset\{\beta\}$. Since $g \in \Lambda_{u}(\mathbb{X})$ we have $s p(g) \subset \Lambda$. Combining these facts we have $s p(g)=\emptyset$, that is, $g=0$.

Now we prove the existence of at least one solution to Eq. (6). For $\Re \lambda \neq 0$, Eq. (6) has a unique solution which is nothing but $\varphi_{f}(\lambda)$. So by definition,

$$
\varphi_{f}(\lambda)=\left(\lambda-\mathcal{D}_{f}\right)^{-1} f, \quad \Re \lambda \neq 0 .
$$

Using a similar argument as in the proof of (iii) of Proposition 2.3 we can show that $\left(\lambda-\mathcal{D}_{f}\right)^{-1} u$ is bounded on $\bar{B}_{r}(i \beta)$ uniformly in $u \in \operatorname{span}\{S(h) f, h \in \mathbb{R}\},\|u\| \leq 1$ for a certain positive constant $r$ independent of $u$ and $\lambda$. Since $i \beta$ is a limit point of $\sigma\left(\mathcal{D}_{f}\right)$, this boundedness yields, in particular, that $i \beta \in \rho\left(\mathcal{D}_{f}\right)$. Hence, there exists a unique solution $g \in \mathcal{M}_{f} \subset \Lambda_{u}(\mathbb{X})$ to (ㅁ).

\section{Almost automorphic functions}

Definition 3.1. A function $f \in C(\mathbb{R}, \mathbb{X})$ is said to be almost automorphic if for any sequence of real numbers $\left(s_{n}^{\prime}\right)$, there exists a subsequence $\left(s_{n}\right)$ such that

$$
\lim _{m \rightarrow \infty} \lim _{n \rightarrow \infty} f\left(t+s_{n}-s_{m}\right)=f(t)
$$

for any $t \in \mathbb{R}$.

The limit in (7) means that

$$
g(t)=\lim _{n \rightarrow \infty} f\left(t+s_{s}\right)
$$

is well-defined for each $t \in \mathbb{R}$ and

$$
f(t)=\lim _{n \rightarrow \infty} g\left(t-s_{n}\right)
$$

for each $t \in \mathbb{R}$. 
Remark 3.2. Because of pointwise convergence the function $g$ is measurable but not necessarily continous. It is also clear from the definition above that constant functions and continuous almost periodic functions are almost automorphic.

If the limit in (8) is uniform on any compact subset $K \subset \mathbb{R}$, we say that $f$ is compact almost automorphic.

Theorem 3.3. Assume that $f, f_{1}$, and $f_{2}$ are almost automorphic and that $\lambda$ is any scalar. Then the following hold true:

(i) $\lambda f$ and $f_{1}+f_{2}$ are almost automorphic,

(ii) $f_{\tau}(t):=f(t+\tau), t \in \mathbb{R}$ is almost automorphic,

(iii) $\bar{f}(t):=f(-t), t \in \mathbb{R}$ is almost automorphic,

(iv) the range $R_{f}$ of $f$ is precompact, and so $f$ is bounded.

Proof. See [21, Theorems 2.1.3 and 2.1.4], for proofs.

Theorem 3.4. If $\left\{f_{n}\right\}$ is a sequence of almost automorphic $\mathbb{X}$-valued functions such that $f_{n} \mapsto f$ uniformly on $\mathbb{R}$, then $f$ is almost automorphic.

Proof. See [21, Theorem 2.1.10], for proof.

Remark 3.5. If we equip $A A(\mathbb{X})$, the space of almost automorphic functions, with the sup norm

$$
\|f\|_{\infty}=\sup _{t \in \mathbb{R}}\|f(t)\|
$$

then it turns out to be a Banach space. If we denote $K A A(\mathbb{X})$, the space of compact almost automorphic $\mathbb{X}$-valued functions, then we have

$$
A P(\mathbb{X}) \subset K A A(\mathbb{X}) \subset A A(\mathbb{X}) \subset B C(\mathbb{R}, \mathbb{X}) .
$$

Theorem 3.6. If $f \in A A(\mathbb{X})$ and its derivative $f^{\prime}$ exists and is uniformly continuous on $\mathbb{R}$, then $f^{\prime} \in A A(\mathbb{X})$.

Proof. See [21, Theorem 2.4.1] for a detailed proof.

Theorem 3.7. Let us define $F: \mathbb{R} \mapsto \mathbb{X}$ by $F(t)=\int_{0}^{t} f(s) d s$ where $f \in A A(\mathbb{X})$. Then $F \in A A(\mathbb{X})$ iff $R_{F}=\{F(t) \mid t \in \mathbb{R}\}$ is precompact.

For any closed subset $\Lambda \subset \mathbb{R}$ we denote by

$$
A A_{\Lambda}(\mathbb{X}):=\left\{u \in A A(\mathbb{X}): s p_{u}(u) \subset \Lambda\right\} .
$$

By the basic properties of uniform spectra of functions, $A A_{\Lambda}(\mathbb{X})$ is a closed subspace of $B C(\mathbb{R}, \mathbb{X})$. Below we denote by $\mathcal{D}_{\Lambda}$ the part of the differential operator $d / d t$ in $A A_{\Lambda}(\mathbb{X})$. Similarly as above we can prove

Lemma 3.8. Under the above notation and assumptions we have

$$
\sigma\left(\mathcal{D}_{\Lambda}\right)=i \Lambda
$$




\section{Almost automorphic solutions}

As a standing assumption in the remaining part of the paper we always assume that $A$ is the infinitesimal generator of an analytic semigroup of linear operators on $\mathbb{X}$. By mild solutions on $\mathbb{R}$ of Eq. (1) we mean continuous solutions to the following equation:

$$
x(t)=T(t-s) x(s)+\int_{s}^{t} T(t-\xi) f(\xi) d \xi, \quad \forall t \geq s, t, s \in \mathbb{R}
$$

where $A$ is the infinitesimal generator of the semigroup $(T(t))_{t \in \mathbb{R}}$ and $f$ is in $A A(\mathbb{X})$.

4.1. Operators $\mathcal{A}$. Let $\Lambda$ be a closed subset of $\mathbb{R}$. We first consider the operator $\mathcal{A}_{\Lambda}$ of multiplication by $A$ and the differential operator $d / d t$ on the function space $A A_{\Lambda}(\mathbb{X})$. By definition the operator $\mathcal{A}_{\Lambda}$ of multiplication by $A$ is defined on $D\left(\mathcal{A}_{\Lambda}\right):=\left\{g \in A A_{\Lambda}(\mathbb{X}): g(t) \in D(A) \forall t \in \mathbb{R}, A g(\cdot) \in A A_{\Lambda}(\mathbb{X})\right\}$, and $\mathcal{A} g:=A g(\cdot)$ for all $g \in D\left(\mathcal{A}_{\Lambda}\right)$.

Lemma 4.1. Assume that $\Lambda \subset \mathbb{R}$ is closed. Then the operator $\mathcal{A}_{\Lambda}$ of multiplication by $A$ in $A A_{\Lambda}(\mathbb{X})$ is the infinitesimal generator of an analytic $C_{0}$-semigroup on $A A_{\Lambda}(\mathbb{X})$.

Proof. We will prove that $\mathcal{A}_{\Lambda}$ is a sectorial operator on $A A_{\Lambda}(\mathbb{X})$. In fact, first we check that $\mathcal{A}_{\Lambda}$ is densely defined. Consider the semigroup $\mathcal{T}_{\Lambda}(t)$ of operators of multiplication by $T(t)$ on $A A_{\Lambda}(\mathbb{X})$. We now show that it is strongly continuous. Indeed, suppose that $g \in A A_{\Lambda}(\mathbb{X})$. Since $R(g)$ is relatively compact (see [21]) we see that the map $[0,1] \times \overline{R(g)} \ni(t, x) \mapsto T(t) x \in \mathbb{X}$ is uniformly continuous. Hence,

$$
\sup _{s \in \mathbb{R}}\|T(t) g(s)-g(s)\| \rightarrow 0
$$

as $t \rightarrow 0$, i.e., the $\mathcal{T}_{\Lambda}(t)$ are strongly continuous. By definition, $g \in D\left(\mathcal{A}_{\Lambda}\right)$ if and only if $g(s) \in D(A), \forall s \in \mathbb{R}$ and $A g(\cdot) \in A A_{\Lambda}(\mathbb{X})$. Thus,

$$
\frac{T(t) g(s)-g(s)}{t}=\frac{1}{t} \int_{0}^{t} T(\xi) A g(s) d \xi, \quad \forall t \geq 0, s \in \mathbb{R} .
$$

Therefore,

$$
\lim _{t \rightarrow 0^{+}} \sup _{s \in \mathbb{R}}\left\|\frac{T(t) g(s)-g(s)}{t}-\frac{1}{t} \int_{0}^{t} T(\xi) A g(s) d \xi\right\|=0,
$$

i.e., $g$ is in $D(G)$, where $G$ is the generator of $\mathcal{T}_{\Lambda}(t)$ and $\mathcal{A}_{\Lambda} g=G g$. Conversely, we can easily show that $G \subset \mathcal{A}_{\Lambda}$.

Now it suffices to prove that $\sigma\left(\mathcal{A}_{\Lambda}\right) \subset \sigma(A)$ to claim that $\mathcal{A}_{\Lambda}$ is a sectorial operator. In fact, let $\mu \in \rho(A)$. To prove that $\mu \in \rho\left(\mathcal{A}_{\Lambda}\right)$ we show that for each $h \in A A_{\Lambda}(\mathbb{X})$ the equation $\mu g-\mathcal{A}_{\Lambda} g=h$ has a unique solution in $A A_{\Lambda}(\mathbb{X})$. But this follows from the fact that $\left(\mu-\mathcal{A}_{\Lambda}\right)^{-1} h(\cdot) \in A A_{\Lambda}(\mathbb{X})$ and that the equation $\mu x-A x=y$ has a unique solution $x$ in $\mathbb{X}$ for any $y \in \mathbb{X}$.

Theorem 4.2. Let $A$ be the generator of an analytic semigroup. Then the operator $\mathcal{A}_{\Lambda}$ of multiplication by $A$ and the differential operator $\mathcal{D}_{\Lambda}$ on $A A_{\Lambda}(\mathbb{X})$ are commuting and satisfy condition $P$ (for the definition see the Appendix). 
Proof. By the above lemma the operator $\mathcal{A}_{\Lambda}$ is sectorial. It suffices to show that it commutes with the differential operator $\mathcal{D}_{\Lambda}$. In fact, since $1 \in \rho\left(\mathcal{D}_{\Lambda}\right)$ we will prove that

$$
R\left(1, \mathcal{D}_{\Lambda}\right) R\left(\omega, \mathcal{A}_{\Lambda}\right)=R\left(\omega, \mathcal{A}_{\Lambda}\right) R\left(1, \mathcal{D}_{\Lambda}\right),
$$

for sufficiently large real $\omega$. Since $\mathcal{A}_{\Lambda}$ generates the semigroup $\mathcal{T}_{\Lambda}(t)$, using wellknown facts from the semigroup theory, the above identity for sufficiently large $\omega$ is equivalent to the following:

$$
R\left(1, \mathcal{D}_{\Lambda}\right) \int_{0}^{\infty} e^{-\omega t} \mathcal{T}_{\Lambda}(t) d t=\int_{0}^{\infty} e^{-\omega t} \mathcal{T}_{\Lambda}(t) d t R\left(1, \mathcal{D}_{\Lambda}\right) .
$$

In turn, (12) follows from the following:

$$
R\left(1, \mathcal{D}_{\Lambda}\right) \mathcal{T}_{\Lambda}(\tau)=\mathcal{T}_{\Lambda}(\tau) R\left(1, \mathcal{D}_{\Lambda}\right), \quad \forall \tau \geq 0,
$$

which is obvious.

So, by the spectral properties of sums of commuting operators, we have

Corollary 4.3. If $\sigma(A) \cap i \Lambda=\oslash$, then for every $f \in A A_{\Lambda}(\mathbb{X})$ there exists a unique $u \in A A_{\Lambda}(\mathbb{X})$ such that

$$
\overline{\mathcal{A}_{\Lambda}+\mathcal{D}_{\Lambda}} u=f .
$$

Proof. Since $\mathcal{A}_{\Lambda}$ and $\mathcal{D}_{\Lambda}$ commute and satisfy Condition $\mathrm{P}$, the sum $\mathcal{A}_{\Lambda}+\mathcal{D}_{\Lambda}$ is closable (denote its closure by $\left.\overline{\mathcal{A}_{\Lambda}+\mathcal{D}_{\Lambda}}\right)$. From $\sigma(A) \cap i \Lambda=\oslash$ and Theorem 5.3 in the Appendix, it turns out that $0 \in \rho\left(\overline{\mathcal{A}_{\Lambda}+\mathcal{D}_{\Lambda}}\right)$. Therefore for every $f \in A A_{\Lambda}(\mathbb{X})$ there exists a unique $u \in D\left(\overline{\mathcal{A}_{\Lambda}+\mathcal{D}_{\Lambda}}\right)$ such that

$$
\overline{\mathcal{A}_{\Lambda}+\mathcal{D}_{\Lambda}} u=f \text {. }
$$

Now our remaining task is just to explain what the above closure means. More precisely, we will relate it with the notion of mild solutions to evolution equations.

Lemma 4.4. Let $u, f \in A A(\mathbb{X})$. If $u \in D\left(\overline{\mathcal{A}_{\Lambda}+\mathcal{D}_{\Lambda}}\right)$ and $\overline{\mathcal{A}_{\Lambda}+\mathcal{D}_{\Lambda}} u=f$, then $u$ is a mild solution of $E q$. (1).

Proof. This lemma follows immediately from the following: For every $u \in A A(\mathbb{X})$ we say it belongs to $D(L)$ of an operator $L$ acting on $A A(\mathbb{X})$ if there is a function $f \in A A(\mathbb{X})$ such that

$$
u(t)=T(t-s) u(s)+\int_{s}^{t} T(t-\xi) f(\xi) d \xi, \quad \forall t \geq s, t, s \in \mathbb{R} .
$$

By a similar argument as in the proof of [15. Lemma 3.1] we can prove that $L$ is a closed single-valued linear operator acting on $A A(\mathbb{X})$ that is an extension of $\mathcal{A}_{\Lambda}+\mathcal{D}_{\Lambda}$. Thus, $L$ is an extension of $\overline{\mathcal{A}_{\Lambda}+\mathcal{D}_{\Lambda}}$. This yields that $u$ is a mild solution of Eq. (11).

As an immediate consequence of the above argument we have:

Theorem 4.5. Let $A$ be the generator of an analytic semigroup, and let $\Lambda$ be a closed subset of $\mathbb{R}$. Then it is necessary and sufficient for each $f \in A A_{\Lambda}(\mathbb{X})$ that there exists a unique mild solution $u \in A A_{\Lambda}(\mathbb{X})$ to Eq. (11) such that the condition $\sigma(A) \cap i \Lambda=\oslash$ holds. 
Proof. The sufficiency follows from the above argument. The necessity can be shown as follows: for every $\xi \in \Lambda$, obviously the function $h: \mathbb{R} \ni t \mapsto a e^{i \xi t}$ is in $A A_{\Lambda}(X)$, where $a \in \mathbb{X}$ is any given element. By assumption, there is a unique $g \in D\left(\mathcal{A}_{\Lambda}\right)$ such that $i \xi g(t)-A g(t)=h(t)$ for all $t \in \mathbb{R}$. Following the argument in [15, p. 252] one can easily show that $g(t)$ is of the form $b e^{i \xi t}$. Hence, $b$ is the unique solution of the equation $i \xi b-A b=a$. That is, $i \xi \notin \sigma\left(\mathcal{A}_{\Lambda}\right)$, and so $i \Lambda \cap \sigma\left(\mathcal{A}_{\Lambda}\right)=\oslash$.

Corollary 4.6. Let $A$ be the generator of an analytic semigroup such that $\sigma(A) \cap$ $i s p_{u}(f)=\oslash$. Then Eq. (11) has a unique almost automorphic mild solution $w$ such that $s p_{u}(w) \subset s p_{u}(f)$.

Proof. Set $\Lambda=s p_{u}(f)$. Then by the above argument we get the theorem.

Remark 4.7. We notice that all results stated above for almost automorphic solutions hold true for compact almost automorphic solutions if the assumption on the almost automorphy of $f$ is replaced by the compact almost automorphy of $f$. Details of the proofs are left to the reader.

\section{Appendix: Sums of COMmuting Operators}

We recall now the notion of two commuting operators, which will be used in the sequel.

Definition 5.1. Let $A$ and $B$ be operators on a Banach space $G$ with nonempty resolvent set. We say that $A$ and $B$ commute if one of the following equivalent conditions holds:

(i) $R(\lambda, A) R(\mu, B)=R(\mu, B) R(\lambda, A)$ for some (all) $\lambda \in \rho(A), \mu \in \rho(B)$,

(ii) $x \in D(A)$ implies $R(\mu, B) x \in D(A)$ and $A R(\mu, B) x=R(\mu, B) A x$ for some (all) $\mu \in \rho(B)$.

For $\theta \in(0, \pi), R>0$ we denote $\Sigma(\theta, R)=\{z \in \mathbf{C}:|z| \geq R,|\arg z| \leq \theta\}$.

Definition 5.2. Let $A$ and $B$ be commuting operators. Then

(i) $A$ is said to be of class $\Sigma(\theta+\pi / 2, R)$ if there are positive constants $\theta, R$ such that $0<\theta<\pi / 2$, and

$$
\Sigma(\theta+\pi / 2, R) \subset \rho(A) \quad \text { and } \sup _{\lambda \in \Sigma(\theta+\pi / 2, R)}\|\lambda R(\lambda, A)\|<\infty ;
$$

(ii) $A$ and $B$ are said to satisfy condition $P$ if there are positive constants $\theta, \theta^{\prime}, R, \theta^{\prime}<\theta$ such that $A$ and $B$ are of class $\Sigma(\theta+\pi / 2, R)$ and $\Sigma\left(\pi / 2-\theta^{\prime}, R\right)$, respectively.

If $A$ and $B$ are commuting operators, $A+B$ is defined by $(A+B) x=A x+B x$ with domain $D(A+B)=D(A) \cap D(B)$.

In this paper we will use the following norm, defined by $A$ on the space $\mathbf{X}$ : $\|x\|_{\mathcal{T}_{A}}:=\|R(\lambda, A) x\|$, where $\lambda \in \rho(A)$. It is seen that different $\lambda \in \rho(A)$ yield equivalent norms. We say that an operator $C$ on $\mathbf{X}$ is $A$-closed if its graph is closed with respect to the topology induced by $\mathcal{T}_{A}$ on the product $\mathbf{X} \times \mathbf{X}$. It is easily seen that $C$ is $A$-closable if $x_{n} \rightarrow 0, x_{n} \in D(C), C x_{n} \rightarrow y$ with respect to $\mathcal{T}_{A}$ in $\mathbf{X}$ implies $y=0$. In this case, $A$-closure of $C$ is denoted by $\bar{C}^{A}$. 
Theorem 5.3. Assume that $A$ and $B$ commute. Then the following assertions hold:

(i) If one of the operators is bounded, then

$$
\sigma(A+B) \subset \sigma(A)+\sigma(B) .
$$

(ii) If $A$ and $B$ satisfy condition $P$, then $A+B$ is $A$-closable, and

$$
\sigma\left(\overline{(A+B)}^{A}\right) \subset \sigma(A)+\sigma(B) .
$$

In particular, if $D(A)$ is dense in $\mathbf{X}$, then $\overline{(A+B)}^{A}=\overline{A+B}$, where $\overline{A+B}$ denotes the usual closure of $A+B$.

Proof. For the proof we refer the reader to [1, Theorems 7.2, 7.3].

\section{REFERENCES}

1. W. Arendt, F. Räbiger, A. Sourour, Spectral properties of the operator equation AX + XB = Y, Quart. J. Math. Oxford (2), 45 (1994), 133-149. MR 95g:47060

2. W. Arendt, C.J.K. Batty, M. Hieber, F. Neubrander, Vector-valued Laplace transforms and Cauchy problems, Monographs in Mathematics, 96, Birkhäuser Verlag, Basel, 2001. MR 2003g:47072

3. B. Basit, Harmonic analysis and asymptotic behavior of solutions to the abstract Cauchy problem, Semigroup Forum 54 (1997), 58-74. MR 98f:47049

4. C.J.K. Batty, W. Hutter, F. Räbiger, Almost periodicity of mild solutions of inhomogeneous periodic Cauchy problems, J. Differential Equations 156 (1999), 309-327. MR 2001c:34116

5. G. Da Prato, P. Grisvard, Sommes d'opérateurs linéaires et équations différentielles opérationelles, J. Math. Pures Appl. 54 (1975), 305-387. MR 56:1129

6. E.B. Davies, "One-parameter Semigroups", Academic Press, London, 1980. MR 82i:47060

7. . T. Diagana and G. M. N'Guerekata, Some remarks on almost automorphic solutions of some abstract differential equations, Far East J. of Math. Sci. 8(3) (2003), 313-322. MR 2004d:34123

8. K.J. Engel, R. Nagel, One-parameter Semigroups for Linear Evolution Equations. Springer, Berlin, 1999. MR 2000i:47075

9. J.A. Goldstein, Semigroups of Linear Operators and Applications, Oxford Mathematical Monographs, Oxford University Press, Oxford, 1985. MR 87c:47056

10. Y. Hino, S. Murakami, Almost automorphic solutions for abstract functional differential equations. Preprint.

11. Y. Hino, T. Naito, N.V. Minh, J.S. Shin, Almost Periodic Solutions of Differential Equations in Banach Spaces. Taylor \& Francis, London - New York, 2002. MR 2003i:34115

12. R. Johnson, A linear almost periodic equation with an almost automorphic solution, Proc. Amer. Math. Soc. 82 (1981), no. 2, 199-205. MR 82i:34044a

13. Y. Katznelson, An Introduction to Harmonic Analysis, Dover Publications, New York, 1976. MR 54:10976

14. B.M. Levitan, V.V. Zhikov, Almost Periodic Functions and Differential Equations, Moscow Univ. Publ. House 1978. English translation by Cambridge University Press, 1982. MR 84g:34004

15. S. Murakami, T. Naito, N.V. Minh, Evolution semigroups and sums of commuting operators: a new approach to the admissibility theory of function spaces, J. Differential Equations 164 (2000), 240-285. MR 2001d:47063

16. T. Naito, N.V. Minh, Evolution semigroups and spectral criteria for almost periodic solutions of periodic evolution equations, J. Differential Equations 152 (1999), 358-376. MR 99m:34131

17. T. Naito, N.V. Minh, J. S. Shin, New spectral criteria for almost periodic solutions of evolution equations, Studia Mathematica 145 (2001), 97-111. MR 2002d:34092

18. T. Naito, Nguyen Van Minh, J. Liu, On the bounded solutions of Volterra equations, Applicable Analysis. To appear.

19. J. M. A. M. van Neerven, The Asymptotic Behavior of Semigroups of Linear Operators, Birkhäuser, Basel, 1996. MR 98d:47001 
20. G. M. N'Guerekata, Almost automorphic functions and applications to abstract evolution equations, Contemporary Math. 252 (1999), 71-76. MR 2001a:34097

21. G. M. N'Guerekata Almost Automorphic and Almost Periodic Functions in Abstract Spaces, Kluwer, Amsterdam, 2001. MR 2003d:43001

22. A. Pazy, Semigroups of Linear Operators and Applications to Partial Differential Equations, Applied Math. Sci. 44, Springer-Verlag, Berlin-New York 1983. MR 85g:47061

23. J. Prüss, Evolutionary Integral Equations and Applications, Birkhäuser, Basel, 1993. MR 94h:45010

24. W. M. Ruess, W.H. Summers, Almost periodicity and stability for solutions to functionaldifferential equations with infinite delay, Differential and Integral Equations, 9 (1996), no. 6, 1225-1252. MR 97m:34159

25. W.M. Ruess, Q.P. Vu, Asymptotically almost periodic solutions of evolution equations in Banach spaces, J. Differential Equations 122 (1995), 282-301. MR 96i:34143

26. W. Shen, Y. Yi, Almost Automorphic and Almost Periodic Dynamics in Skew-Product Semiflows, Memoirs of the Amer. Math. Soc. 136 (1998). MR 99d:34088

27. Q.P. Vu, E. Schüler, The operator equation $A X-X B=C$, admissibility, and asymptotic behaviour of differential equations, J. Differential Equations 145 (1998), 394-419. MR 99h:34081

28. S. Zaidman, Topics in abstract differential equations, Pitman Research Notes in Mathematics Series, 304, Longman Scientific \& Technical, New York, 1994. MR 95f:34087

Department of Mathematics, Howard University, 2441 6th Street N.W., Washington D.C. 20059

E-mail address: tdiagana@howard.edu

Department of Mathematics, Morgan State University, 1700 E. Cold Spring Lane, Baltimore, Maryland 21251

E-mail address: gnguerek@jewel.morgan.edu

Department of Mathematics, Hanoi University of Science, Khoa Toan, Dai Hoc Khoa Hoc Tu Nhien, 334 Nguyen Trai, Hanoi, Vietnam

E-mail address: nvminh@netnam.vn

Current address: Department of Mathematics, State University of West Georgia, Carrollton, Georgia 30118

E-mail address: ngvminh@yahoo.com 